\title{
Impact of Mechanical Thrombectomy on the Organization of the Management of Acute Ischemic Stroke
}

\author{
Benoît Daubail ${ }^{a, b}$ Frédéric Ricolfi ${ }^{a, c}$ Pierre Thouant ${ }^{a, c}$ Charlotte Vogue ${ }^{a, c}$ \\ Adrien Chavent $^{\mathrm{a}, \mathrm{c}}$ Guy-Victor Osseby ${ }^{\mathrm{a}, \mathrm{b}}$ Marie Hervieu-Begue ${ }^{\mathrm{a}, \mathrm{b}}$ \\ Benoit Delpont $^{a, b}$ Bruno Mangola ${ }^{d}$ Yannick Bejot ${ }^{a, b}$ Maurice Giroud ${ }^{a, b}$

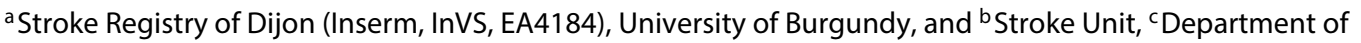 \\ Interventional Neuro-Radiology, and ${ }^{d}$ Emergency Wards, Dijon University Hospital, Dijon, France
}

\section{Key Words}

Ischemic stroke · Fibrinolysis - Endovascular treatment .

Stent retriever $\cdot$ Stroke networks

\begin{abstract}
Background: Several trials and meta-analyses have recently demonstrated the superiority of endovascular therapy over standard medical treatment in patients presenting with acute ischemic stroke. In order to offer the best possible treatment to a maximum number of patients, many stroke care networks probably have to be reorganized. After analyzing the reliability of data in the literature, an algorithm is suggested for a pre-hospital and in-hospital alert system to improve the timeliness of subsequent treatment: a drip-andship approach. Summary: Five recent well-designed randomized studies have demonstrated the benefit of endovascular therapy associated with intravenous fibrinolysis by recombinant tissue plasminogen activator (rt-PA) for acute ischemic stroke with confirmation by recent meta-analyses. The keys for success are a very short time to reperfusion, within $6 \mathrm{~h}$, a moderate to severe pre-treatment deficit ( $\mathrm{Na}$ tional Institute of Health around 17), cerebral imaging able to identify proximal large vessel occlusion in the anterior cir-
\end{abstract}

culation, a limited infarct core and a reversible penumbra, the use of the most recent devices (stent retriever) and a procedure that avoids general anesthesia, which reduces blood pressure. To meet these goals, every country must build a national stroke infrastructure plan to offer the best possible treatment to all patients eligible for intravenous fibrinolysis and endovascular therapy. The plan may include the following actions: inform the population about the first symptoms of stroke, provide the call number to improve the timeliness of treatment, increase the number of comprehensive stroke centers, link these to secondary and primary stroke centers by telemedicine, teach and train paramedics, emergency doctors and radiologists to identify the stroke infarct, proximal large vessel occlusion and the infarct core quickly, train a new generation of endovascular radiologists to improve access to this therapy. Key Message: After 20 years of rt-PA, this new evidence-based therapy is a revolution in stroke medicine that will benefit patients. However, a new robust and multi-disciplinary care strategy is necessary to transfer the scientific data into clinical practice. It will require reorganization of the stroke infrastructure, which will include comprehensive stroke centers and secondary and primary stroke centers. The winners will be patients with severe stroke.

(c) 2016 S. Karger AG, Basel

\section{KARGER}

E-Mail karger@karger.com

www.karger.com/ene
(C) 2016 S. Karger AG, Basel

0014-3022/16/0752-0041\$39.50/0
Professor Maurice Giroud

Stroke Unit

CHU Dijon-Bourgogne - University Hospital of Dijon

1 Bd Jeanne d'Arc, FR-21000 Dijon (France)

E-Mail maurice.giroud@chu-dijon.fr 


\section{Introduction}

Ischemic stroke is a major public health problem [1]. Intravenous recombinant tissue plasminogen activator (rt-PA) administered within $4.5 \mathrm{~h}$ after symptom onset in patients below 80 years has up to now been the only proven reperfusion therapy for acute cerebral ischemia [2,3].

As well as having several limitations with regard to indications and contraindications, it has been shown to be largely ineffective in opening thromboembolic occlusions in the carotid and basilar arteries and in the first segment of the middle cerebral artery (MCA) [4]. rt-PA achieves recanalization in only one-third of patients with a proximal occlusion, and the prognosis without recanalization is poor [5]. Finally, local administration of rt-PA or urokinase into the clot has been evaluated in several studies without really convincing results [6].

Mechanical endovascular thrombectomy mechanical treatment (MT) was the next logical step [7], initially with the MERCI retrieval systems followed by the PENUMBRA aspiration systems and then by stent retrievers, notably the SOLITAIRE. After 3 negative trials published in 2013 [8-10], MT with stent retrievers was shown to be safe and effective in 5 recent trials published in 2015 [11-16].

This review aims to analyze the results of the last endovascular trials [11-16] and the recent meta-analyses [17-20] corresponding to almost $15 \%$ of the patients treated by rt-PA, as well as the organizational issues, namely the implementation of a system that includes triaging patients with suspected stroke in primary stroke centers and their transport toward comprehensive stroke centers as quickly as possible. Decision-making would be facilitated by the use of algorithms for MT and telemedicine. Other major goals include providing information to the public and the training of professionals.

\section{Material and Methods}

The Lessons of the Latest Endovascular Trials [8-16] and Meta-Analyses [17-20] (Table 1)

The initial clinical trials of MT for acute ischemic stroke published in 2013 (SYNTHESIS [8], MR RESCUE [9], IMS III [10]; table 1) failed to show a significant benefit of MT, because these trials had several limitations including biases in cerebral and vascular imaging and in use of rt-PA and less effective mechanical devices, all of which contributed to heterogeneity in the results. Knowledge of these factors contributed to improvements in the following trials. After the publication of the first positive results of the MR CLEAN study $[11,12]$, 4 other endovascular trials in stroke care have demonstrated the benefit of endovascular treatment, associated or not with intravenous fibrinolysis: ESCAPE [13], EX-
TEND-IA [14], SWIFT-PRIME [15] and REVASCAT [16] (table 1). Subgroups analyses [17-20] showed that functional outcomes were significantly better in the following conditions:

- Thrombectomy within $6 \mathrm{~h}$ after stroke onset

- Angiographic imaging that confirmed proximal arterial occlusion $(\mathrm{p}<0.001)$

- Patients who received the combined therapy $(p=0.018)$

- When stent retriever devices were used for MT $(\mathrm{p}<0.001)$

- The selection of patients based on a severe neurological deficit with an National Institute of Health Stroke Scale (NIHSS) score around 17

- A mean delay of 85-127 min between first symptom onset and rt-PA

- The best results observed in patients with a large penumbra area and numerous collaterals $[13,14,17-20]$

- The treatment of the great majority of patients without general anesthesia

The overall results showed a global benefit of rt-PA and MT since only 3-7 patients needed to be treated to avoid one death or severe handicap at 3 months.

\section{Results}

The Goals of Optimal Organization [21-24]

It is important to insist on the following goals:

- Offer this bi-therapy to a maximum number of patients. This target is important because almost $10 \%$ of ischemic stroke patients are eligible for rt-PA, and 15\% of stroke patients eligible for rt-PA may benefit from $\mathrm{MT}$. These patients will thus require rt-PA $+\mathrm{MT}$, and it will be necessary to lead to reorganize stroke care systems in every country to meet these goals.

- Provide a maximum of patients with timely access to bi-therapy, by reducing pre- and intra-hospital time delays, which are a major concern in acute stroke care.

\section{The Tools to Reach These Objectives}

This will be the most difficult part of the programme:

- Every Health Ministry must develop a public health policy around the new management of stroke, by providing financial resources to create a maximum number of stroke units.

- A greater number of interventional neuro-radiologists must be trained. The question of training vascular neurologists in interventional radiology must be studied.

- Telestroke ambulances in pre-hospital stroke management and mobile stroke units with CT scan and biological kits and connected to tele-stroke centers may be a solution in rural regions [25].

- The population at large and paramedics in particular must be aware of the first symptoms of stroke through information on the FAST test.
Daubail et al. 
Table 1. Descriptive features of patients included in the randomized trials (from references [8-20])

\begin{tabular}{|c|c|c|c|c|c|c|c|c|}
\hline $\begin{array}{l}\text { SYNTHESIS [8], } \\
2013\end{array}$ & $<6$ & 100 & $0(0)$ & 66.0 & 13 & $\begin{array}{l}148 \\
\text { NA } \\
225\end{array}$ & $0.84(0.55-1.27)$ & $1.52(0.80-2.88)$ \\
\hline IMS III [10], 2013 & $<5$ & CT (46.6) & $434(100)$ & 69.0 & 17 & $\begin{array}{l}\text { NS } \\
122.4 \\
208\end{array}$ & $1.11(0.79-1.55)$ & $0.86(0.58-1.28)$ \\
\hline $\begin{array}{l}\text { MR CLEAN [12], } \\
2015\end{array}$ & $<6$ & CT, CTA (100) & $203(87)$ & 65.8 & 17 & $\begin{array}{r}204 \\
85 \\
260\end{array}$ & $2.05(1.36-3.09)$ & $0.94(0.61-1.44)$ \\
\hline $\begin{array}{l}\text { EXTEND-IA [14], } \\
2015\end{array}$ & $<6$ & $\begin{array}{l}\text { CT, CTA } \\
\text { CTP (100) }\end{array}$ & $35(100)$ & 68.6 & 17 & $\begin{array}{l}\text { NS } \\
127 \\
210\end{array}$ & $3.75(1.38-10.17)$ & $0.38(0.09-1.59)$ \\
\hline $\begin{array}{l}\text { SWIFT-PRIME [15], } \\
2015\end{array}$ & $<6$ & $\begin{array}{l}\text { CT, CTA or } \\
\text { MRI (100) }\end{array}$ & $98(100)$ & 65.0 & 17 & $\begin{array}{l}190.5 \\
110.5 \\
224\end{array}$ & $2.75(1.53-4.94)$ & $0.72(0.29-1.79)$ \\
\hline $\begin{array}{l}\text { REVASCAT [16], } \\
2015\end{array}$ & $<8$ & CT, CTA (100) & $70(68)$ & 65.7 & 17 & $\begin{array}{l}223 \\
117.5 \\
269\end{array}$ & $1.98(1.11-3.53)$ & $1.23(0.59-2.55)$ \\
\hline
\end{tabular}

NA = Not applicable; NS = not specified

The Technical Aspects to Resolve

What Imaging?

The objective is to perform both cerebral parenchyma and vascular imaging by either $\mathrm{CT}$ scan with perfusion (CTP) and CT angiography (CTA) or by MRI, magnetic resonance diffusion and perfusion and magnetic resonance angiography $[17,19,26]$.

ESCAPE [13], EXTEND-IA [14] and SWIFT PRIME [15] included sophisticated imaging techniques to identify patients with large areas of salvageable brain tissue since such patients have a greater chance of benefiting from rt-PA + MT. The ESCAPE [13] study evaluated collateral circulation using multiphase CTA, the EXTEND-IA [14] trial used perfusion imaging, and the SWIFT PRIME [15] study started off with a similar protocol to EXTEND-IA [14] and later switched to the protocol used by ESCAPE [13]. The meta-analyses [17-20] demonstrated the great consistency between the clinical populations enrolled, the 2 imaging procedures used depending on the human and logistics resources $[17,19$, 26].

Badhiwala et al. [18] found that patients who did not undergo CTA to confirm the presence of a proximal occlusion did not benefit from MT. The primary stroke centers should be able to perform CTA and CTP, thus allowing early perfusion of intravenous rt-PA by tele-stroke to save time before moving the patient toward a comprehensive stroke center for MT according to the drip-and-ship approach $[18,19]$.

\section{Place of Previous rt-PA?}

In ESCAPE, 27\% of the patients who underwent endovascular therapy did not receive rt-PA; the proportion was $13 \%$ in MR CLEAN and 32\% in REVASCAT. A similar benefit of clot removal was observed in the 2 groups (rt-PA alone and in rt-PA + MT). The meta-analysis of 
Badhiwala et al. [18] included the 8 trials with both positive and negative primary outcomes published since 2013. The main finding from this meta-analysis was that the proportion of patients attaining functional independence was markedly greater in those who underwent endovascular treatment associated with prior intravenous rt-PA: $31 \%$ in those receiving rt-PA alone and $45 \%$ in those given endovascular therapy as well $(\mathrm{p}=0.005)$. This was provided without any major safety hazards with similar rates of intracranial hemorrhage and all-cause mortality in the 2 groups. The early start of rt-PA may reduce clot burden and facilitate arterial recanalization by MT $[18,19]$.

Possible Negative Role of General Anesthesia

A new subgroup analysis of the MR CLEAN trial [12] demonstrated that outcomes in patients treated with endovascular therapy with general anesthesia $(n=79)$ were not as good as those in patients treated with medical therapy alone. The odds of reduced disability modified Rankin Scale (mRS) with endovascular therapy was 2.13 (1.46$3.11)$ with local anesthesia versus $1.09(0.69-1.71)$ with general anesthesia when compared with patients with no endovascular therapy. The odds of functional independence (mRS score of $0-2)$ was 2.79 (1.70-4.59) for local anesthesia versus 1.09 (0.56-2.12) for general anesthesia, compared with patients with no endovascular therapy. In ESCAPE [13] and EXTEND-IA [14], less than $10 \%$ of patients had general anesthesia. Thus, the results of both trials are only applicable to patients with local anesthesia and there is no evidence of the benefit of thrombectomy in patients treated under general anesthesia. General anesthesia induces a decrease in blood pressure, which may aggravate ischemic lesions.

Nevertheless, even for local anesthesia, the anesthetist must be present. This may change when the results of ongoing randomized clinical trials comparing local with general anesthesia are known.

\section{Role of Pre-Hospital Care}

Given these positive results, the key challenge is to reorganize the system to achieve an early start of rt-PA and make MT available to all eligible patients [27-29], by restructuring stroke care networks dedicated to thrombolysis so as to incorporate this new technology [17].

In 2015, Sheppard et al. [22] evaluated the relationship between time to the request for CT, time to first contact with the stroke team, the recording of onset time, stroke identification using the FAST test and sending of a prealert message. The study highlighted the importance of pre-alerting the hospital, accurate stroke identification and recording the onset time in making the right decision in acute stroke. Patients whose stroke had not been recognized in the pre-hospital setting had a high risk of being excluded from comprehensive treatment. To change this situation, patients and their entourage must be aware of the first symptoms of stroke using the FAST test and then the inter-hospital organization must be able to deliver rt$\mathrm{PA}$ and MR directly in a tertiary comprehensive center or via tele-stroke for rt-PA perfusion [27-29].

\section{Role of the Professional Training}

It will necessary to train general practitioners, emergency doctors, neurologists, radiologists and emergency ward nurses as previously demonstrated [30]. This professional training, focused on stroke triage, will depend on the size of the stroke team, which often depends on the urban or rural location of the unit. It will be necessary to increase the number of stroke units, the number of neurologists and in particular the number of endovascular neuro-radiologists, all of whom will be included in a stroke network.

This medical progress in terms of functional outcome is only possible if the organization of care in a region is optimal allowing the access to this specific therapy to a maximum number of patients [17-20, 23, 31].

\section{Proposal for a Stroke Infrastructure Dedicated to}

rt-PA + MT: A Drip-and-Ship Approach [27-29]

Every hospital performing rt-PA must be able to detect large vessel occlusion by CTA. If not available, the hospital's imaging capacity must be developed. Technically, CTA can be performed with the great majority of today's CT scanners. The major issue, however, is the limited availability of qualified personnel $24 / 7$. This problem can be circumvented by setting up tele-radiology along with tele-stroke $[25,30]$.

Depending on the imaging findings, the patient will then be managed in a dedicated stroke unit using rt-PA alone or transferred under bridging therapy to a comprehensive stroke center offering endovascular therapy [21, 23 ]. In urban regions with good coverage by comprehensive stroke centers, patients with high NIHSS scores $(>10)$ who have a high probability of large vessel occlusions should primarily be directed to centers that provide endovascular neuro-radiology services [23].

The following algorithm may be very useful to meet the expectations of the public (fig. 1):

- A stroke center equipped with a stroke unit managed by neuro-vascular experts and interventional neuro-
Daubail et al. 
Fig. 1. Algorithm for transport decisions

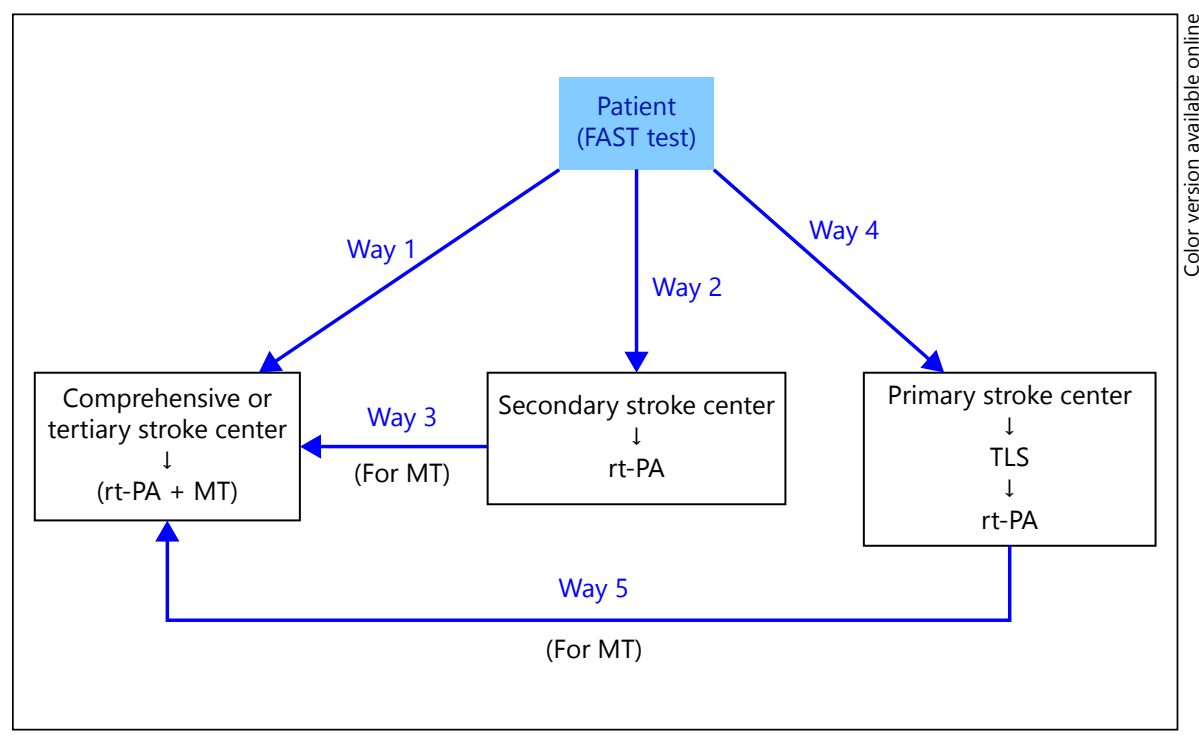
according to stroke infrastructure.

Table 2. Eligibility criteria for both rt-PA and MT

\begin{tabular}{ll}
\hline rt-PA & MT \\
\hline Inclusion criteria & \\
Clinical symptoms & \\
$\quad$ Ischemic stroke & - Ischemic stroke with CTA demonstration of MCA and/or ICA occlusion \\
$\quad$ NIHSS score $<25$ & - Within $6 \mathrm{~h}$ \\
$\quad$ For $3-4.5 \mathrm{~h}$ window & - Aspects score $>6$ or mismatch on perfusion imaging \\
\hline Exclusion criteria & \\
- Hemorrhagic stroke & Also \\
- >One-third MCA territory infarct up to 4.5 h window & Also \\
- History of nontraumatic intracranial hemorrhage & Also \\
- Subarachnoid hemorrhage & Also \\
- Blood pressure $>185 / 110$ & Also \\
- Major surgery with bleeding risk within 14 days & Also \\
- Major head trauma & Possible \\
- Vitamin K antagonist with INR $>1.7$ & Possible \\
- Direct oral anticoagulant without reversal & Possible \\
- Platelets $<100,000$ & Possible \\
\hline
\end{tabular}

radiologists will be the comprehensive or tertiary stroke center to treat any patient (way 1 ).

- A secondary stroke center with no interventional radiologist, but included in a tele-stroke network, will perform the rt-PA (way 2) and will then move the patient to the comprehensive stroke center for MT (way 3 ).

- A patient first managed in a primary stroke center (way 4) can undergo rt-PA by the use of tele-stroke with the comprehensive stroke center and will then be moved to the comprehensive stroke center (way 5).

Thrombectomy in Ischemic Stroke

\section{Proposal for Eligibility Criteria for Combined rt-PA} and MT (Table 2)

Following the synthesis provided by the meta-analysis $[18,19]$ and the current American Heart Association and American Stroke Association guidelines [19, 29], rt-PA must be administered to all eligible patients (table 2) within $0-3 \mathrm{~h}$ (Class I, level A), within the 3-4.5 h delay (Class I, level B). Efforts should be made to reduce delays to reperfusion therapy, thus leading to better clinical outcomes (Class I, level A). The guidelines [17, 19, 29] recommend stent retrievers rather than earlier generation 
coil retrievers (Class I, level A). Guidelines [17] should recommend MT within the first $6 \mathrm{~h}$ as a level-1 evidencebased treatment in acute ischemic stroke, in patients with proximal carotid and/or MCA occlusion confirmed by neuro-vascular imaging and with respect to exclusion criteria (table 2).

The following criteria are used to identify the ideal patient for MT, some criteria are recognized (table 2): age, ASPECT score, NIH score, time to treatment, demonstration of a proximal artery occlusion (internal carotid artery or MCA) [19].

Time from stroke onset to recanalization and reperfusion will be reduced if the public is informed about the symptoms of stroke and paramedics and emergency physicians are trained to identify candidate patients who may benefit from MT, so that neuro-interventional resources can be mobilized quickly.

\section{Conclusion}

It is now certain that endovascular therapy must be systematically used in patients presenting with ischemic stroke with proximal large artery occlusion within $6 \mathrm{~h}$ after the first symptoms [17-20].
To deal with high volumes and to reduce delays, a stroke infrastructure must be developed in every country, according to a defined algorithm that allows appropriate transport decisions for the ideal clinical case, which would be a patient with large vessel occlusions and an NIHSS score of 10 or more.

Further studies will be necessary to evaluate this strategy in routine practice in every country and to evaluate the balance between benefits, costs and service efficiency, with the aim to optimize treatment for these patients.

For all health professionals, this is an exciting new challenge for the organization of pre-hospital and in-hospital stroke care [24-29].

\section{Acknowledgments}

We thank the University Hospital of Dijon, the University of Burgundy, the Regional Stroke Network, Dijon Stroke Registry (Inserm, InVS) and Philip Bastable for reviewing the English version of the article.

\section{Disclosure Statement}

The authors report no conflicts of interest.

\section{References}

1 Giroud M, Jacquin A, Béjot Y: The worldwide landscape of stroke in the 21 st century. Lancet 2014;383:195-197.

2 Tissue plasminogen activator for acute ischemic stroke. The national institute of neurological disorders and stroke rt-PA stroke study group. N Engl J Med 1995;333:15811587.

3 Jauch EC, Saver JL, Adams HP Jr, et al: Guidelines for the early management of patients with acute ischemic stroke: a guideline for healthcare professionals from the American Heart Association/American Stroke Association. Stroke 2013;44:870-947.

4 Riedel CH, Zimmermann P, Jensen-Kondering $U$, et al: The importance of size: successful recanalization by intravenous thrombolysis in acute anterior stroke depends on thrombus length. Stroke 2011;42:1775-1777.

5 Lima FO, Furie KL, Silva GS, et al: Prognosis of untreated strokes due to anterior circulation proximal intracranial arterial occlusions detected by use of computed tomography angiography. JAMA Neurol 2014;71:151-157.

6 Ogawa A, Mori E, Minematsu K, et al: Randomized trial of intraarterial infusion of urokinase within 6 hours of middle cerebral artery stroke: the middle cerebral artery embo- lism local fibrinolytic intervention trial (MELT) Japan. Stroke 2007;38:2633-2639.

7 Mazighi M, Serfaty JM, Labreuche J, et al: Comparison of intravenous alteplase with a combined intravenous-endovascular approach in patients with stroke and confirmed arterial occlusion (RECANALISE study): a prospective cohort study. Lancet Neurol 2009; 8:802-809.

8 Ciccone A, Valvassori L, Nichelatti M, et al; SYNTHESIS Expansion Investigators: Endovascular treatment for acute ischemic stroke. N Engl J Med 2013;368:904-913.

9 Kidwell CS, Jahan R, Gornbein J, et al; MR RESCUE Investigators: A trial of imaging selection and endovascular treatment for ischemic stroke. N Engl J Med 2013;368:914-923.

10 Broderick JP, Palesch YY, Demchuk AM, et al; Interventional Management of Stroke (IMS) III Investigators: Endovascular therapy after intravenous $\mathrm{t}-\mathrm{PA}$ versus $\mathrm{t}-\mathrm{PA}$ alone for stroke. N Engl J Med 2013;368:893-903.

11 Fransen PS, Beumer D, Berkhemer OA, et al; MR CLEAN Investigators: MR CLEAN, a multicenter randomized clinical trial of endovascular treatment for acute ischemic stroke in The Netherlands: study protocol for a randomized controlled trial. Trials 2014;15:343.
12 Berkhemer OA, Fransen PS, Beumer D, et al; MR CLEAN Investigators: A randomized trial of intraarterial treatment for acute ischemic stroke. N Engl J Med 2015;372:11-20.

13 Goyal M, Demchuk AM, Menon BK, et al ESCAPE Trial Investigators: Randomized assessment of rapid endovascular treatment of ischemic stroke. N Engl J Med 2015;372: 1019-1030.

14 Campbell BC, Mitchell PJ, Kleinig TJ, et al; EXTEND-IA Investigators: Endovascular therapy for ischemic stroke with perfusionimaging selection. N Engl J Med 2015;372: 1009-1018.

15 Saver JL, Goyal M, Bonafe A, et al; SWIFT PRIME Investigators: Stent-retriever thrombectomy after intravenous t-PA vs. t-PA alone in stroke. N Engl J Med 2015;372:22852295.

16 Jovin TG, Chamorro A, Cobo E, et al; REVASCAT Trial Investigators: Thrombectomy within 8 hours after symptom onset in ischemic stroke. N Engl J Med 2015;372: 2296-2306.

17 Campbell BC, Donnan GA, Lees KR, et al: Endovascular stent thrombectomy: the new standard of care for large vessel ischaemic stroke. Lancet Neurol 2015;14:846-854. 
18 Badhiwala JH, Nassiri F, Alhazzani W, et al: Endovascular thrombectomy for acute ischemic stroke: a meta-analysis. JAMA 2015;314: 1832-1843.

19 Prabhakaran S, Ruff I, Bernstein RA: Acute stroke intervention: a systematic review. JAMA 2015;313:1451-1462.

20 Chen CJ, Ding D, Starke RM, et al: Endovascular vs medical management of acute ischemic stroke. Neurology 2015;85:1980-1990.

21 Spiotta AM, Vargas J, Turner R, et al: The golden hour of stroke intervention: effect of thrombectomy procedural time in acute ischemic stroke on outcome. J Neurointerv Surg 2014;6:511-516.

22 Sheppard JP, Mellor RM, Greenfield S, et al: The association between prehospital care and in-hospital treatment decisions in acute stroke: a cohort study. Emerg Med J 2015;32: 93-99.
23 Jung S, Stapf C, Arnold M: Stroke unit management and revascularisation in acute ischemic stroke. Eur Neurol 2015;73:98-105.

24 Furlan AJ: Endovascular therapy for stroke it's about time. N Engl J Med 2015;372:23472349.

25 Liman TG, Winter B, Waldschmidt C, et al: Telestroke ambulances in prehospital stroke management: concept and pilot feasibility study. Stroke 2012;43:2086-2090.

26 Goyal M, Menon BK, Coutts SB, et al: Effect of baseline CT scan appearance and time to recanalization on clinical outcomes in endovascular thrombectomy of acute ischemic strokes. Stroke 2011;42:93-97.

27 Grotta J, Hacke W: Stroke neurologist's perspective on the new endovascular trials. Stroke 2015;46:1447-1452.
28 Smith EE, Schwamm LH: Endovascular clot retrieval therapy: implications for the organization of stroke systems of care in North America. Stroke 2015;46:1462-1467.

29 Jauch EC, Saver JL, Adams HP Jr, et al: Guidelines for the early management of patients with acute ischemic stroke: a guideline for healthcare professionals from the American Heart Association/American Stroke Association. Stroke 2013;44:870-947.

30 Hervieu-Begue M, Jacquin A, Osseby GV, et al: The role of the clinical nurse within a combined stroke and telefibrinolysis network: the G5 pilot study in Burgundy, France. Eur Res Telemed 2013;2:11-15.

31 Raffe F, Jacquin A, Milleret O, et al: Evaluation of the possible impact of a care network for stroke and transient ischemic attack on rates of recurrence. Eur Neurol 2011;65:239_ 244 